\title{
Associated Factors for Lumbar Degenerative Spondylolisthesis in Japanese Patients with Osteoarthritis of the Hip: A Radiographic Study
}

\author{
Takeshi Sasagawa, Takuya Nakamura \\ Department of Orthopedics Surgery, Toyama Prefectural Central Hospital, Toyama, Japan
}

\section{Study Design: Retrospective study.}

Purpose: To determine the presence and frequency of factors for degenerative spondylolisthesis (DS) in patients with hip osteoarthritis (OA).

Overview of Literature: OA of the hip joint (hip OA) in Japanese patients is associated with a high incidence of degenerative lumbar spondylolisthesis (DS). However the associated factors for DS in patients with hip OA are unclear.

Methods: The study included 518 patients (59 men and 459 women) with a mean age of 63.8 years who underwent total hip arthroplasty for hip OA at our hospital between January 2004 and May 2014. The presence of DS was assessed using preoperative standing lateral radiographs of the hip joint including the lower lumbar spine. To identify the associated factors for DS in patients with hip OA, multiple logistic regression analysis was conducted in which the presence or absence of DS was used as a dependent variable, and age, female sex, body mass index (BMI), sacral slope (SS), Crowe classification, and primary OA (P-OA) not associated with acetabular dysplasia were independent variables.

Results: Patients with DS accounted for 114 (22.0\%) of 518 cases. In multiple logistic regression analysis to identify associated factors for DS in patients with hip OA, the odds ratio for age was 1.05 (95\% confidence interval [CI], 1.02-1.08), female sex was 2.48 (95\% $\mathrm{Cl}, 1.11-5.54)$, BMl was 1.08 (95\% Cl, 1.02-1.14), SS was 1.07 (95\% Cl, 1.04-1.09), Crowe classification was 0.60 (95\% Cl, 0.40-0.91), and $\mathrm{P}-\mathrm{OA}$ was $1.90(95 \% \mathrm{Cl}, 1.20-3.75)$.

Conclusions: Age, female sex, BMI, SS, low Crowe classification, and P-OA are independently associated factors for DS in patients with hip OA.

Keywords: Associated factor; Lumbar degenerative spondylolisthesis; Osteoarthritis of the hip; A radiographic study

\section{Introduction}

Offierski and MacNab [1] defined clinical conditions where flexion contracture and/or leg length inequality based on hip joint disease influenced spinal alignment as hip-spine syndrome. Osteoarthritis of the hip joint (hip $\mathrm{OA}$ ) in Japanese patients is associated with a high incidence of degenerative lumbar spondylolisthesis (DS) [2,3]. It is important to identify associated factors for DS in patients with hip OA to understand its pathology. However,

Received Jan 13, 2016; Revised Feb 8, 2016; Accepted Feb 23, 2016

Corresponding author: Takeshi Sasagawa

Department of Orthopedics Surgery, Toyama Prefectural Central Hospital,

2-2-78 Nishinagae, Toyama 930-8550, Japan

Tel: +81-76-424-1531, Fax:+81-76-422-0667, E-mail: sasagawawagasasa@yahoo.co.jp 
the associated factors for DS in patients with hip OA are unclear. The purpose of the present study was to determine the types and frequency of the associated factors for DS in patients with OA of the hip.

\section{Materials and Methods}

The study included 518 patients ( 59 men and 459 women) with a mean age of 63.8 years (range, 28-91 years) who received total hip arthroplasty for hip OA at our hospital between January 2004 and May 2014. Exclusion criteria were osteonecrosis of the femoral head, trauma, history of osteotomy of hip joint, rheumatoid arthritis, and rapidly destructive coxarthrosis causing rapid and extreme destruction of the hip joint within 6-12 months [4]. This study was approved by Our Institutional Review Board.

Within 3 months before surgery, a frontal radiograph of the hip joint and standing lateral radiographs of the hip joint including the lower lumbar spine were taken. Presence of DS, grade of slip (Meyerding grade) and sacral slope (SS) were accessed. SS was regarded as the angle between the superior endplate of S1 and the horizontal plane. DS was defined as an anterior slip $\geq 3 \mathrm{~mm}$ or more $[2,3,5,6]$. Hip OA was divided into two groups depending on etiology, secondary osteoarthritis (S-OA) that was associated with acetabular dysplasia, and primary osteoarthritis (P-OA) that was not associated with acetabular dysplasia. Acetabular dysplasia was defined as a centeredge angle $<25^{\circ}$. Crowe classification of the grade of hip OA was determined radiologically.

\section{Statistical analyses}

All data are expressed as mean \pm standard deviation. An unpaired Student $t$ and chi-squared tests were used to compare age, sex, body mass index (BMI), SS, the etiology of hip OA (P-OA or S-OA), and Crowe classification between patients in the DS and non-DS group. Demographic data and the relationship between patients in the groups with primary and secondary OA of the hip joint were also assessed. To identify the associated factors for DS in patients with hip OA, multiple logistic regression analysis was conducted in which presence or absence of DS was used as a dependent variable, and age, female sex, BMI, SS, P-OA, and Crowe classification were independent variables. A $p<0.05$ was considered to be statistically significant. All statistical analyses were conducted using
IBM SPSS Statistics for Windows ver. 22 (IBM Corp, Armonk, NY, USA).

\section{Results}

Patients with DS accounted for 114 (22.0\%) of 518 cases. Meyerding grade I and II comprised 105 and 9 cases, respectively. DS was found at L3 in 19 cases, L4 in 89 cases, and L5 in 13 cases (DS was found at two levels in 7 cases). In contrast, patients with spondylolytic spondylolisthesis accounted for just 26 (5.0\%) of 518 cases.

The mean age of patients in the DS and non-DS group was $66.6 \pm 8.7$ years and $63.0 \pm 10.4$ years, respectively. The mean age of patients in the DS group was significantly higher than in the non-DS group $(p<0.01)$. Hip OA occurred in 3 of 33 patients (9.1\%) under 50 years, 17 of 143 patients (11.9\%) in their 50s, 49 of 195 patients (25.1\%) in their 60 s, 39 of 117 patients (33.3\%) in their 70s, and 6 of 30 patients (20.0\%) over 80 years old. Eight of 59 male patients (13.6\%) and 106 of 459 female patients (23.1\%) were associated with DS. There was no significant difference between male and female patients. The average BMI of patients in the DS and non-DS group was $24.5 \pm 4.0$ $\mathrm{kg} / \mathrm{m}^{2}$ and $23.5 \pm 4.0 \mathrm{~kg} / \mathrm{m}^{2}$, respectively. The average BMI of patients in the DS group was significantly higher than that in the non-DS group $(p<0.05)$. The SS in the DS group $\left(37.4^{\circ} \pm 10.2^{\circ}\right)$ was significantly greater than that in the non-DS group $\left(32.9^{\circ} \pm 11.8^{\circ}\right)(p<0.01)$. The prevalence of DS in patients with P-OA and S-OA was 32 of 90 cases (35.6\%) and 82 of 428 cases (19.2\%), respectively. The prevalence of DS in patients with P-OA was significantly higher than that of DS patients with S-OA $(p<0.01)$. The Crowe classification (I/II/III/IV) of hip OA was $100 / 10 / 2 / 2$ in the DS group and 324/43/31/6 in the nonDS group. There was no significant difference between DS and non-DS groups in Crowe classification (Table 1).

The mean age of patients in the P-OA and S-OA group was $71.0 \pm 8.8$ and $62.3 \pm 9.8$ years, respectively. The mean age of patients in the P-OA group was significantly lower than that in the S-OA group $(p<0.01)$. There were no significant group differences in sex and BMI. SS in the S-OA group $\left(34.9^{\circ} \pm 11.0^{\circ}\right)$ was significantly greater than that in the P-OA group $\left(28.9^{\circ} \pm 12.6^{\circ}\right)(p<0.01)$. The prevalence of DS in patients with P-OA was significantly higher than that of DS patients with S-OA $(p<0.01)$. The Crowe classification (I/II/III/IV) of S-OA was significantly greater than that of P-OA group (Table 2). 
Table 1. Demographic data and the relationship between patients in the groups with and without degenerative lumbar spondylolisthesis

\begin{tabular}{lccc} 
Variable & DS group $(\mathrm{n}=114)$ & Non-DS group $(\mathrm{n}=404)$ & $p$-value \\
Age $(\mathrm{yr})$ & $66.6 \pm 8.7$ & $63.0 \pm 10.4$ & $<0.01$ \\
Sex (male/female) & $8 / 106$ & $51 / 353$ & $\mathrm{NS}$ \\
\hline Body mass index $\left(\mathrm{kg} / \mathrm{m}^{2}\right)$ & $24.5 \pm 4.0$ & $23.5 \pm 4.0$ & $<0.05$ \\
$\mathrm{SS}\left(^{\circ}\right)$ & $37.4 \pm 10.2$ & $32.9 \pm 11.8$ & $<0.01$ \\
\hline P-OA/S-OA & $32 / 82$ & $58 / 346$ & $<0.01$ \\
Crowe type (I/II/II/IV) & $100 / 10 / 2 / 2$ & $324 / 43 / 31 / 6$ & $\mathrm{NS}$ \\
\hline
\end{tabular}

NS, not significant in $\chi^{2}$ test; SS, sacral slope; P-OA, primary osteoarthritis; S-OA, secondary osteoarthritis.

Table 2. Demographic data and the relationship between patients in the groups with primary and secondary osteroarthritis of the hip joint

\begin{tabular}{lccc} 
Variable & P-OA group $(\mathrm{n}=90)$ & S-OA group $(\mathrm{n}=428)$ & $p$-value \\
Age $(\mathrm{yr})$ & $71.0 \pm 8.8$ & $62.3 \pm 9.8$ & $<0.01$ \\
Sex (male/female) & $9 / 81$ & $50 / 378$ & $\mathrm{NS}$ \\
\hline Body mass index $\left(\mathrm{kg} / \mathrm{m}^{2}\right)$ & $24.4 \pm 3.9$ & $23.6 \pm 4.1$ & $\mathrm{NS}$ \\
$\mathrm{SS}\left(^{\circ}\right)$ & $28.9 \pm 12.6$ & $34.9 \pm 11.0$ & $<0.01$ \\
\hline DS/non-DS & $32 / 58$ & $82 / 346$ & $<0.01$ \\
Crowe type (I/II/II/IV) & $88 / 1 / 1 / 0$ & $336 / 52 / 32 / 8$ & $<0.01$ \\
\hline
\end{tabular}

P-OA, primary osteoarthritis; S-OA, secondary osteoarthritis; NS, not significant; SS, sacral slope; DS, degenerative spondylolisthesis.

Table 3. Data for each risk factors using multiple logistic regression

\begin{tabular}{lccc} 
Factor & Odds ratio & $95 \%$ Confidence interval & $p$-value \\
\hline Age & 1.05 & $1.02-1.08$ & $<0.01$ \\
Female & 2.48 & $1.11-5.54$ & $<0.05$ \\
\hline BMI & 1.08 & $1.02-1.14$ & $<0.01$ \\
SS & 1.07 & $1.04-1.09$ & $<0.01$ \\
\hline P-OA & 1.90 & $1.20-3.75$ & $<0.05$ \\
Crowe classification & 0.60 & $0.40-0.91$ & $<0.05$ \\
\hline
\end{tabular}

BMI, body mass index; SS, sacral slope; P-OA, primary osteoarthritis.

A multiple logistic regression analysis was done to identify associated factors for DS in patients with hip OA. The odds ratio for age was 1.05 (95\% confidence interval [CI], 1.02-1.08), female sex was 2.48 (95\% CI, 1.11-5.54), BMI was 1.08 (95\% CI 1.02-1.14), SS was 1.07 (95\% CI, 1.041.09), P-OA was 1.90 (95\% CI, 1.20-3.75), and Crowe classification was 0.60 (95\% CI, 0.40-0.91). Age, female sex, BMI, SS, P-OA and low Crowe type were identified as independent associated factor for DS (Table 3).

\section{Discussion}

Although subjects varied in age, sex, symptoms, or inclusion of spondylolytic spondylolisthesis, the prevalence of
DS in Japanese is reported as 3.7\%-12.4\% (Table 4) [58]. In contrast, the prevalence of DS in Japanese patients with hip OA was reported as $31 \%-36 \%$ [2,3]. In the present study, the prevalence of DS in patients with hip OA was $22.0 \%$ (27.0\% when spondylolytic spondylolisthesis is added). This prevalence is consistent with previous reports. Therefore patients with hip OA are associated with a high incidence of DS.

The present study identified age, female sex, BMI, SS, $\mathrm{P}-\mathrm{OA}$, and low Crowe type as independent associated factors for DS in patients with hip OA. The prevalence of DS increases with age [2,5,7-10]. Therefore DS is considered a result of age-related degeneration of the intervertebral disk and facet joints. This tendency was also observed 
Table 4. Prevalence of degenerative lumbar spondylolisthesis in Japanese

\begin{tabular}{lcccl} 
Subjects & No. of subjects & Spondylolisthesis (\%) & Study patients & \multicolumn{1}{c}{ Study } \\
\hline Adults for health check & 5,000 & 3.7 & D & Takahashi et al. [7] \\
Outpatients of orthopedic surgery & 1,728 & 7.1 & D & Fujita et al. [5] \\
\hline Elderly (>65 yr) & 528 & 8.9 & D+S & Horikawa et al. [6] \\
\hline Adult women & 1,245 & 12.4 & D+S & Murata et al. [8] \\
Hip OA & 247 & 30.8 & D & Morimoto et al. [2] \\
Hip OA & 50 & 36.0 & D+S & Kinoshita et al. [3] \\
\hline Hip OA & 518 & 22.0 & D & Present study \\
\hline
\end{tabular}

D, degenerative spondylolisthesis; S, spondylolytic spondylolisthesis; Hip OA, osteoarthritis of hip joint.

presently in hip OA. Patients with hip OA may be associated with a high incidence of DS because the peak age of onset for hip OA and DS is similar.

DS occurred 4-6 times more frequently in women $[9,10]$. This fact has been recognized, but not conclusively explained. In the present study this tendency was also observed in patients with OA of the hip even though the number of male patients might be too small to compare statistically.

Because the prevalence of DS increases with BMI $[10,11], B M I$ of patients in the DS group was significantly higher than that of patients in the non-DS group with hip OA. Being over-weight might lead to an increased axial load on intervertebral disks and facet joints, and it might also lead to an anterior displacement of the trunk and enhance the risk of DS [11].

Schuller et al. [11] and Funao et al. [12] reported that the SS of patients with DS is significantly greater than that of controls $\left(42.3^{\circ}\right.$ vs. $33.4^{\circ}, 34.0^{\circ}$ vs. $26.6^{\circ}$, respectively). In the present study, SS of patients in the DS group was significantly higher than that in patients with hip OA in the non-DS group. If a patient develops a fixed flexion deformity of the hip, the flexion deformity may rotate the pelvis forwards and increase SS. Fixed flexion deformity exaggerates normal lumbar lordosis. Hyperlordosis results in anterior displacement of lumbar vertebrae and may enhance the risk of DS [1].

The present study identified P-OA as an independent associated factor for DS in patients with hip OA even though the SS in the S-OA group was significantly greater than that in the P-OA group. To our knowledge, no prior studies have examined the possible epidemiological relationship between the etiology of hip OA (P-OA or S-OA) and DS. Low Crowe type was identified as an independent associated factor. Considerable P-OA is also associated strongly with DS compared with S-OA, because in this study 92 of 94 patients (97.9\%) in Crow type II/III/IV had S-OA. Hip OA is considered to be influenced by age, genetics [13], and environment [14]. There seems to be a common predisposition for a tendency for degeneration between $\mathrm{P}-\mathrm{OA}$, in which OA changes occur even without acetabular dysplasia, and DS, which is a result of degeneration of intervertebral disks and facet joints.

In the present study, we assessed various parameters using preoperative standing lateral radiographs of the hip joint including the lower lumbar spine. Limitations of the present study include a lack of consideration of the range of motion of the hip joint, alignment of the entire spine, lumbar lordosis, and facet joint orientation. Although pelvic incidence is an important morphologic parameter correlated with susceptibility to pathomechanisms responsible for the onset and progression of DS [12], we could not assess it because deformity and high dislocation of femoral head exist in many cases with hip OA, especially secondary OA that accounts for the majority of hip $\mathrm{OA}$ in Japan. Further study is necessary to understand the pathogenesis of DS in hip OA more fully.

\section{Conclusions}

Age, female sex, BMI, SS, P-OA, and low Crowe type are independent associated factors for DS in patients with hip OA.

\section{Conflict of Interest}

No potential conflict of interest relevant to this article was reported. 


\section{References}

1. Offierski CM, MacNab I. Hip-spine syndrome. Spine (Phila Pa 1976) 1983;8:316-21.

2. Morimoto T, Nishida K, Shigematsu M, Sonohata M, Mawatari M, Hotokebuchi T. Association of hip osteoarthritis with lumbar degenerative spondylolisthesis: hip-spine syndrome. Rinshoseikeigeka 2008; 43:683-7.

3. Kinoshita M, Washimi M, Kataoka O, Sho T, Den M. Radiological analysis of lumbar spine in hip disorders; correlation to the instability of lumbar spine. Rinshoseikeigeka 1992;27:933-7.

4. Postel M, Kerboull M. Total prosthetic replacement in rapidly destructive arthrosis of the hip joint. Clin Orthop Relat Res 1970;72:138-44.

5. Fujita M, Matsuda Y, Kawatani Y, Ogata N, Sokabe $\mathrm{H}$, Yamamoto H. Evaluation and frequency of anterolisthesis and retrolisthesis in lumbar spine: a radiographic survey. J Chugoku-Shikoku Orthop Assoc 2003;15:271-5.

6. Horikawa K, Kasai Y, Yamakawa T, Sudo A, Uchida A. Prevalence of osteoarthritis, osteoporotic vertebral fractures, and spondylolisthesis among the elderly in a Japanese village. J Orthop Surg (Hong Kong) 2006;14:9-12.

7. Takahashi S, Terayama K, Otsuka K, Wada K, Kinoshita H. An epidemiological survey on spondy- lolisthesis of the lumbar spine using the X-rays of health-check services. Chubuseisaishi 1990;33:72830.

8. Murata H, Kushida K, Machida A, Inoue T. Epidemiologic study of lumbar spondylolisthesis in female inhabitants. J Lumbar Spine Disord 1998;4:36-41.

9. Rosenberg NJ. Degenerative spondylolisthesis: predisposing factors. J Bone Joint Surg Am 1975;57:46774 .

10. Jacobsen S, Sonne-Holm S, Rovsing H, Monrad H, Gebuhr P. Degenerative lumbar spondylolisthesis: an epidemiological perspective: the Copenhagen Osteoarthritis Study. Spine (Phila Pa 1976) 2007;32:120-5.

11. Schuller S, Charles YP, Steib JP. Sagittal spinopelvic alignment and body mass index in patients with degenerative spondylolisthesis. Eur Spine J 2011;20:7139.

12. Funao H, Tsuji T, Hosogane N, et al. Comparative study of spinopelvic sagittal alignment between patients with and without degenerative spondylolisthesis. Eur Spine J 2012;21:2181-7.

13. Mototani H, Mabuchi A, Saito S, et al. A functional single nucleotide polymorphism in the core promoter region of CALM1 is associated with hip osteoarthritis in Japanese. Hum Mol Genet 2005;14:1009-17.

14. Yoshimura N, Sasaki S, Iwasaki K, et al. Occupational lifting is associated with hip osteoarthritis: a Japanese case-control study. J Rheumatol 2000;27:434-40. 